\title{
SPECIALIST AND ADVANCED SPECIALIST NURSING AND MIDWIFERY PRACTICE
}

Prof Sinegugu Duma: University of Cape Town,

Ms Joan Dippenaar: University of Limpopo Medunsa campus,

Prof Busi Bhengu: University of KwaZulu Natal,

Prof Annamart Oosthuizen: UNISA,

Dr Lyn Middleton: University of KwaZulu Natal,

Ms Maria Phillips: University of the Free State,

Ms Susan Naude: University of Limpopo Medunsa campus,

Prof Leana R Uys: FUNDISA

\begin{abstract}
Ambiguities and role confusion exist with regard to specialist and clinically advanced nursing/midwifery practice globally and in most healthcare settings. This confusion requires clarification in such a way that specialist/clinical advanced nursing and midwifery practice (as a category of the clinical specialist) are clearly delineated. In South Africa, this is further complicated by the introduction of the Occupation Specific Dispensation (OSD) of the Department of Health (2007) which makes provision for a grade called Professional Nurse (Specialty Nursing) and also for a further grade called Clinical Nurse Specialist (Level 3 Hospitals).

The Specialty Nursing category has been implemented in most health services and nurses who have qualifications at post-basic and postgraduate diploma level in the areas described in Regulation 212 can benefit from this progression. The higher level of Clinical Nurse Specialist (OSD term for advanced specialist) has not yet been implemented. The OSD document indicates that appointments to these posts will require significant clinical experience and a clinical master's degree. This is in line with the ICN stance that clinical advanced specialist nursing and midwifery practice require masters and PhD-level education linked to significant clinical practice experience.

This chapter presents a differentiation between the two levels of specialist practice - the advanced nurse/midwife practitioner and the specialist nurse/midwife from a South African perspective and in line with the International Council of Nurses (ICN) framework.
\end{abstract}

Keywords: Distance learning; Education; Nursing

\section{Introduction}

Ambiguities and role confusion exist with regard to specialist and clinically advanced nursing/midwifery practice globally and in most healthcare settings. This confusion requires clarification in such a way that specialist/clinical advanced nursing and midwifery practice (as a category of the clinical specialist) are clearly delineated. Since 2002, the International Council of Nurses (ICN) has developed and published the definition, nature, scope, role and functions of the advanced and specialist nurse/midwife practitioner as a guideline for role development. Differentiation is made between two levels of specialist practice - the advanced nurse/midwife practitioner and the specialist nurse/midwife. South African nurses/midwives were co-developers and co-authors of these documents. 
In 2010 the SA Nursing Council (SANC) asked FUNDISA to prepare a discussion document on the nurse specialist and advanced specialist. FUNDISA supports the development of a framework to guide the depth, breadth and range of clinical and non-clinical specialist nursing/midwifery knowledge, theory, experience and research particular to the South African healthcare context as proposed by the Higher Education framework. The regulation of nursing practice and education is needed to ensure consistent, high-quality, safe and accessible healthcare to the public. Several frameworks for nurse/midwifery specialists exist globally. These reflect the context for which they were developed and may not fit the needs of the South African healthcare context.

FUNDISA's views, as presented in this document, are aligned with the principles, guidelines and framework of the International Council of Nurses (ICN) that describe the competencies of the nurse/midwifery specialist (ICN regulation Series 2009) to be used as a guideline for role development of nursing and midwifery practice, in a quest for a suitable framework for South Africa. Midwifery practice is considered alongside nursing in this document. FUNDISA's proposals were formally adopted by SANC on the 28-29th of March 2012 and will be communicated as such in a circular i.e. the specialist nurse/midwife and advanced specialist/midwife.

The clinical pathway for nursing and midwifery specialist practice, whether at one or more levels, is seen as a strategy for improved healthcare service delivery. The technical report of the WHO (1996) on nursing practice found that specialist nurses/midwives contribute considerably to the improvement of healthcare quality and services, specifically in poorly serviced areas. Nurses/midwives are often the only caregivers to the poor.

To facilitate understanding, the following terms are used consistently in this chapter: nurse/midwifery specialist for the first level of nurse specialist, and advanced specialist nurse/midwife for the second level.

\section{International background}

Specialist nursing/midwifery is universally associated with post basic courses/programmes that evolved in the $20^{\text {th }}$ century to improve access to, and quality of, healthcare service delivery in a specific area of practice (World Health Organization, WHO 1996). An extensive ICN review (2009: 5) found no uniformity in the title, scope of practice, education, standards of practice and paths of entry of specialist/advanced nurse/midwifery practices. This lack of standardization has led to ambiguities, differences in standards and confusion around the definition and role of specialist/advanced nurses/midwives. For example, the Canadian Framework for Advanced Nurse Practice (2008) mainly refers to specialist nurse practice without recognition of advanced practice (clinicians) as suggested by the ICN (2006).

In 1992 the ICN initiated a revision of the nurse/midwifery specialisation (2009: 5). This resulted in the development of criteria and standards for the education, practice and regulation of specialist nurse/midwifery practice.

\section{The South African Healthcare Context Historical background}

The first educational programmes preparing advanced nurse practitioners were developed in the 1950s as post-basic diplomas (du Preez, 1988). The first postgraduate advanced programme, a Master's degree in Advanced Intensive Care, was developed at the University of Pretoria in 1970. Du Preez, a nurse manager at the Groote Schuur hospital, found in an analysis of the development of specialist educational programmes that they went hand-in-hand with the development of medical 
science and technology, and also made medical advances possible. For instance, the Diploma in Operating Theatre Nursing commenced about eight years before the first organ transplants were done in this hospital and the first intensive care units were set up.

Advanced nurse practitioners were introduced to South African health services in a "relatively uncontrolled" manner according to du Preez, who was for many years the chief Matron of the Groote Schuur Hospital and lived through many of these changes (1988). Usually no additional or special posts were created, but nurses were identified and trained and used in a specialist capacity without regard for their formal position. Nurse managers found them to be a valuable resource, and were willing to use "generalist" posts for "specialist nurses". At the time that these advanced practice roles developed, the work in general hospitals was to a large extent still done by student nurses, with registered nurse supervising and teaching. The specialist or advanced practice role enabled registered nurses to "return" to a clinical role and, in the areas where they functioned, they became excellent role models for students and projected the image of nursing that is valued by the profession and the community.

When a new career -specific dispensation for nursing was created in early 1982, it had three levels of professional nurses (Professional Nurse PN, Senior Professional Nurse SPN, Chief Professional Nurse CPN). The idea was that this should be a CLINICAL career ladder, since the administrative ranks started with a Nursing Service Manager (Bruwer, 1988). However, the more senior ranks were quickly involved in management responsibilities, and the CPN and CPN became a "zone matron" or a "section head" or "unit manager", and little came of the clinical practice expert. Usually, nurses still had to become managers or educators to gain promotion.

In 1985 the directives for nursing courses/programmes that lead to additional qualifications were amended by the South African Nursing Council (SANC). These are still valid in 2010. The SANC Regulation 212 guides the practice and education of 14 formal post-basic nursing and midwifery programmes (SANC Reg. 212). The names of these courses were changed from "advanced" to "postbasic" nursing programmes in 1999 (Penn-Kekana \& Blaauw 2003:19) and are mainly post-basic diplomas that lead to additional qualifications registered by the SANC. A recent study (Dippenaar et al, 2009) indicated that $66 \%$ of post-basic midwifery training (2009) was offered by Universities in South Africa, mainly as post-basic diplomas. These programmes can all be considered as "specialist" programmes.

While a formal system of licensure, registration, and certification to support and manage these nurse/midwifery practice categories is in place through the SANC, there is no scope of practice for this group of practitioners as yet. The pathway to the additional professional qualification may be through a diploma or a clinical honours' or master's degree programme (the latter is offered at the higher or tertiary education level).

The accreditation of a professional qualification by the SANC does not make a distinction between a diploma and a degree when the additional qualification is registered.

In addition a variety of informal short courses and in-service programmes exist to build capacity in nurses and midwives to meet the healthcare services' needs. These are offered through many institutions, including non-governmental organizations (NGOs), for example the initiation of Antiretroviral Therapy by Nurses (Johns Hopkins Program for International Education In Gynecology and Obstetrics JHPIEGO 2008), and the Department of Health (DoH) for nurses to provide termination of pregnancy services. Most of these programmes and courses aim at health service provision rather than career development of nurses, without formal recognition or remuneration. However, several areas of practice specialisation have not been formally acknowledged as 
qualifications (for example stoma care, wound care and childbirth education). There is at present no research database of the type of courses and the number of practitioners in these particular fields. The absence of a clear framework of education and practice makes it very difficult to evaluate and establish additional practice areas as formal specialities and qualifications.

\section{Current and future options}

Currently there is no differentiation in the professional qualifications between specialist and advanced specialist nursing and midwifery practice. Formal recognition and clinical career paths in the healthcare structures in South Africa are underdeveloped when compared with the ICN's guidelines.

The Occupation Specific Dispensation (OSD) of the Department of Health (2007) makes provision for a grade called Professional Nurse (Specialty Nursing) and also for a further grade called Clinical Nurse Specialist (Level 3 Hospitals). In the Dispensation, career progression is linked to educational qualification as well as clinical experience and, as the title implies, the monetary reward is linked to the work that the nurse is currently employed to do (occupation specific). This means that there is no additional remuneration for academic qualifications not clearly related to what the nurse is currently employed to do. In effect this means that an academic qualification in nursing education will not reap a remuneration benefit for a nurse running a clinical oncology unit. The Specialty Nursing category has been implemented in most health services and nurses who have qualifications at post-basic and postgraduate diploma level in the areas described in Regulation 212 can benefit from this progression.

The higher level of Clinical Nurse Specialist (OSD term for advanced specialist) has not yet been implemented. The OSD document indicates that appointments to these posts will require significant clinical experience and a clinical master's degree. This is in line with the ICN stance that clinical advanced specialist nursing and midwifery practice require masters and PhD-level education linked to significant clinical practice experience.

The new Higher Education Qualifications Framework (2007) and South African Qualification Authority (SAQA) make provision for specialist/advanced nursing/midwifery practice with consideration of the developmental status of the South African population. A professional B degree or an Advanced Diploma in nursing is considered as a future entry point for specialist/advanced nurse/midwifery practice according to the new proposed nursing qualifications framework. Accordingly, all professional nursing education falls within the Higher Education band in South Africa.

\section{Nurse/Midwife Specialist Definition}

A nurse/midwife specialist (NS or MS) is a professional person who has been prepared beyond the level of a generalist and is authorised to practise as a specialist in a branch of the nursing/midwifery field. This includes clinical, teaching, administration, research and consultancy (adapted from ICN 2009: 6).

A specialist nurse/midwife practitioner is a registered nurse/midwife clinician who has acquired the expert clinical knowledge and skills that include complex decision-making abilities and clinical competencies for expanded practice, the characteristics of which are shaped by the context and/or country in which she/he has the credentials to practice. The advanced nurse/midwife practitioner is a clinical specialist who has the appropriate credentials to practice autonomously. 


\section{Criteria designating nurse/midwifery specialities}

The following criteria are considered essential and underpin the nurse/midwifery speciality practice (ICN, 2009). Specialist nurse and midwifery practice needs to:

- $\quad$ practise as a nurse;

- $\quad$ adhere to the foundational registration of a professional nurse;

- be sufficiently complex and advanced beyond the scope of a general professional nurse;

- exist due to a demand and need for the speciality;

- focus on a recurring problem that lies within the nursing discipline;

- be based on a core body of nursing/midwifery knowledge, refined by research;

- have established educational and practice standards;

- have expertise obtained through approved professional advanced educational programmes administered by a nurse/midwife;

- have financial and human resources available to support the programme accredited by the profession; and

- ensure that specialist practitioners are organised and represented in interest groups/associations/societies.

\section{Advanced Nurse or Midwife Specialist}

\section{Definition}

An advanced nurse/midwife specialist (ANS or AMS) is a specialist clinician with a broad autonomous practice managing a specific case load. This advanced nurse/midwife specialist may function as first entry-point and needs the knowledge and expertise to be able accurately to assess, diagnose and manage the patient population in the speciality area. This may include being able to make a "medical diagnosis" and prescribe treatment. This usually requires expertise in diagnostic testing and treatment beyond the normal practice of the nurse/midwife. In South Africa she/he may practise as a private practitioner, but this is not exclusive to this category.

\section{Criteria designating advance nurse/midwifery specialities}

The advanced nurse/midwifery specialist needs to (ICN, 2009):

- use advanced clinical assessment and decision-making;

- function with a high degree of autonomy and independence;

- manage a specified caseload;

- implement a range of advanced clinical competencies;

- integrate research, education and clinical management in practice;

- provide consultancy to other health professionals;

- be recognized as a first point of entry of service;

- advocate for patient's rights and improved evidence-based quality of care;

- develop best practice in a specialty field (evidence based practice);

- participate in policy development for improved practice;

- publish based on practice and practise research; and

- manage a business or project. 


\section{Scope of Practice}

The scope of practice defines the accountabilities of the role occupant. Currently there is no published scope of practice guideline for specialist/advanced nurses/midwives in South Africa, but a process is underway to develop this. The role and functions of a specialist or advanced specialist nurse/midwife are determined by the scope of practice, responsibilities and activities for which the registration/licensed professional is educated and authorised within a healthcare system (ICN 2009: 6). This is specific to the speciality and may differ, depending on the contexts. The following aspects need consideration in the development of the scope of practice of specialist and advanced nurse practitioners:

- Contextual realities: The availability of healthcare workers, ratios and skill-mix, financial resources, categories of skill shortages, poor or underserviced areas, and infrastructure are factors that will influence the categories of specialist and advanced nurse/midwife practitioners needed .

- The level of independence/autonomy of advanced/specialist nurse/midwife practice: A high degree of professional autonomy and independence of practice is required in specialists and advanced specialist practice which is facilitated by the legal and structural framework.

- Development of a formal system of licensure, registration, and certification: A legal framework is needed to recognize standards for competency-based specialist and advanced specialist nursing and midwifery education and practice though a collaborative process involving all stakeholders, including the SANC, DOH, and clinical nursing experts.

- Educational preparation: Annexures $2 \& 3$ of this document reflect the guidelines of the content of the existing educational frameworks for the educational preparation of the specialist and advanced nurse/midwifery programmes as presented by the ICN and other frameworks. These can be used as a point of departure for South Africa. It indicates the essential competencies required for each role.

\section{Competencies}

The competencies of a specialist/advanced nurse/midwife are firmly based on the basic foundational competencies of a generalist nurse/midwife (ICN 2009: 7). These basic foundational competencies are reflected in the new scope of practice of nurses and midwives (in progress) for South Africa. It is the ICN's (2009: 9) position that the authority to practise as a registered professional nurse/midwife is an essential requirement and entry level for specialist practice.

The competency framework (as reflected in Annexures $1 \& 2$ ) has been developed by the ICN as a guideline for specialist/advanced nurse and midwifery practice and needs to be refined and adapted for the South African healthcare context by all stakeholders. Twelve (12) competencies have been identified for a specialist and 8 domains for the advanced practitioner.

\section{Education and Training}

In South Africa there are currently a number of qualifications which lead to registration as a specialist nurse. These are: postgraduate or post-basic diploma programmes, post-basic Bachelor's degrees, clinical Honours' degrees and Master's degrees. After numerous discussions and consideration of international experience and publications, as well as the current South African context including national policies and directions in health care provision and higher education, FUNDISA agreed on a set of guiding principles and recommendations in 2011. University Nursing schools were encouraged to follow these guidelines in their planning. 
- The academic qualification for becoming a nurse/midwifery specialist should be at Postgraduate Diploma level;

- The academic qualification for becoming an advanced nurse/midwife specialist should be at Master's degree level. This should be a clinical master's and should follow on the Postgraduate Diploma and direct entry from a Bachelor's degree should not be allowed.

The following suggestions about the preparation of nurse specialists and advanced nurse specialists were prepared by the Education Portfolio Committee of FUNDISA in 2012, based on the National Qualifications Framework as stipulated in Higher Education Legislation, as well as the SA Nursing Council's framework.

\section{The Postgraduate Diploma in Nursing (PGD)}

The PGD should be focused on preparing a specialist competent to practise at a specialist level. The programme will be a minimum of 120 credits, and will include a substantial amount of course and clinical work. A credit represents 10 notional hours of student activity, as defined in the SAQA documents (SAQA, 2000, p.23).

Course work:

- Core: not more than $42 \%$ of credits should be dedicated to this component. Although the core is the same for all specialties, it should be applied within the student's own specialty area. The core includes topics such as professional practice and ethics, the Primary Health Care approach, clinical leadership, interpersonal skills for professionals, use and interpretation of statistics, literature review, utilization of published research and data related to healthcare and practice.

- Specialty and specialty practice: At least $58 \%$ should be dedicated to this component. The speciality practice components include an evidence base of practice in the discipline field intentionally linked to best practice, refined competencies linked to assessment, planning, doing or delegating care of the patient group and their families in the SA context of care, practice-monitoring and evaluation.

This programme is not aimed at preparing researchers, but students should be taught how to access research and how to evaluate it for implementation in practice. The preparation for this competency is seen as part of the core component.

The suggestion is that at least 4 hours of clinical role-taking practice should be included in the programme for every 1 credit. That means that for a 120 credit programme, 480 hours of clinical practice would be included. These hours are not part of the 120 credits but are aimed at developing the student into a confident and competent nurse specialist and should be linked to providing evidence of clinical competence.

\section{The professional Master's in Nursing degree (MN)}

The professional Master's degree should be focused on preparing an advanced nurse specialist who is proficient to practise at this level. The advanced nurse specialist is part of the clinical career ladder and is different from a career path in management or education. The programme will be a minimum of 180 credits and will include a substantial amount of clinical practice. The students should in effect spend most of their time linked to a functional multidisciplinary clinical team in a variety of clinical practice settings where they can learn and establish their contribution to practise as advanced nurse/midwife specialists. 
This qualification is modelled on the registrar model as practised in medicine, with the specialist trained by working closely with a consultant and team in clinical practice. FUNDISA therefore recommends the creation of "registrar posts" for nurses doing a professional Master's degree, so that they can be salaried, but dedicated to contributing to service provision while practising as advanced nurse/midwife specialists in a service and training role.

Research should form at least $50 \%$ of the content of this programme. However, it should be strongly practice-focused and projects should be chosen so that they are relevant to improving clinical practice.

Course work should not be heavily classroom-based, but should address developing clinical expertise and current practice, programme management, consultancy, and shifting practice to an evidencebased model. It is strongly recommended that this qualification and the course work be multiprofessional in nature.

The summative evaluation should include an authentic, comprehensive, practice-based examination.

\section{Recognizing New Specialties}

Many speciality areas (like multiple sclerosis, Parkinson's disease and geriatrics) are additional internationally identified areas of specialist nurse practice in health care. In some healthcare systems, up to 60 specialist areas are recorded. Only seventeen (17) specialist areas, leading to additional qualifications, are identified in the Higher Education Framework (ETQA, SAQA) and by the SANC for South Africa. These could be extended to include already developed areas, such as forensic and neonatal nursing.

There is currently no formal process for the SA Nursing Council to recognize new specialties in nursing. The Occupation Specific Dispensation, as discussed earlier, can only recognise clinical specialties deemed to be such by the Council, but the Council has not approved new specialties for some time.

FUNDISA's position is that the Council should have regulations that prescribe the process and criteria for such recognition. The process recommended by FUNDISA is as follows:

- An application for a new specialty to be introduced has to be put forward by a specialist organization representing the specialty group.

- The application should address and be evaluated, based on the following criteria:

- There is a need for the specialty in the health services;

- Positions for such nurse specialists have been created in the health services;

- There is a body of knowledge and a set of competencies which allows for a 120 credit course in the specialty area;

- There are enough people who can teach this specialty in South Africa.

- Once a new specialty has been approved, NEls can submit educational programmes to train such specialists.

If is important for the development of the profession and for the delivery of quality health services that the profession is allowed to grow appropriate specialties. Medicine already recognizes more than 30 specialty areas, and some super-specialty areas. Such development is part of the growth of modern health services and nursing in this country is lagging behind. 


\section{Conclusion}

This chapter outlines a policy proposal that FUNDISA has submitted to the SA Nursing Council with some amendments, and for which it is lobbying in appropriate forums. It is important for the profession that we make our own decisions on specialist and advanced specialist practice in this country. There is no doubt that the additional training and education received by these specialists makes a difference to healthcare delivery. It also helps the professional nurses to develop and grow, and therefore keeps them engaged and dedicated. As Higher Education Institutions, the Universities are particularly dedicated to producing professionals with a high level of professional skills.

\section{References}

ADVANCED NURSE PRACTICE, A NATIONAL FRAMEWORK. CANADIAN NURSES ASSOCIATION. 2008 Ottowa. www.cna-aiic.ca

Bruwer, A. 1988. The Clinical Nursing Specialist in South Africa: financial considerations. In Dewar, S. R. (ed.) The Clinical Nursing Specialist in Southern Africa: Seminar and Workshop Proceedings. Durban: University of Natal, School of Nursing.

Dippenaar, J M, Sengane, M.L.M, Erasmus, D. \& Digamale, R. 2009. Scorecard for midwifery in Higher Education. Presentation for ICN Durban.

DOH. 2009. Nursing strategy for South Africa. Pretoria.

Department of Health 2007. Occupation Specific Dispensation ()SD) Professional Nurse. Pretoria: $\mathrm{DOH}$.

Du Preez, L. J. 1988. The Clinical Nurse Specialist in South Africa: an administrative perspective. In Dewar, S. R. (ed.) The Clinical Nursing Specialist in Southern Africa: Seminar and Workshop Proceedings. Durban: University of Natal, School of Nursing.

ICN POSITITON STATEMENT 2002. Participation of Nurses in Health Services Decision Making and Policy Development. Available www.icn Accessed 17/1/2009).

INTERNATIONAL COUNCIL OF NURSES. 2009. ICN Framework of Competencies for Nurse Specialists, ICN Regulation Series. Geneva.

SAQA 2000. The National Qualifications Framework and Curriculum Development. Pretoria: SAQA.

Penn-Kekana, L. \& Blaauw, D. 2003. A rapid appraisal of maternal health services in the South African health care system. Health System Development DFID (Department of International Development):151.

\section{SANC 1981}

World Health Organization. 1996. Nursing Practice. Report of a WHO Expert Committee. World Health Organization. Geneva. 


\section{Annexure 1}

Competency framework for specialist nurse/midwife (ICN 2009: 10-30)

The following competencies are considered for specialist nursing and midwifery practice. Similarities exist and speciality knowledge is specific for each speciality

\section{- Accountability}

Knowledge and skills in addition to those acquired for practice as a registered nurse.

- Specialised scope of practice;

- Specific codes of practice (involving the multidisciplinary team);

- Shared competency, responsibility and accountability;

- Professional supervision within legal and institutional policies and protocols.

- Ethical practice

Knowledge and skills in addition to those acquired for practice as a registered nurse.

- Contemporary issues specific to the speciality;

- Approaches to ethical decision-making, analysis and proposing solutions;

- Advocacy strategies;

- Human and health rights' instruments for the context of care applicable to the speciality;

- Confidentiality and privacy specific to speciality;

- Concepts of society, customs, beliefs and values specific to speciality;

- Knowledge and practices and beliefs of ethnic/religious groups in speciality, and adapting practices to meet ethnic/religious beliefs.

- Communication

- Food

- Personal hygiene

- Physical contact

- Family

- Religion

- Health practices

- Strategies to deal with harmful traditional practices;

- Safety of practice (environment) specific to speciality area.

- Legal practice

Knowledge and skills in addition to those acquired for practice as a registered nurse.

- Laws relevant to speciality practice area;

- Professional regulation of speciality area;

- Codes/rules/regulation specifically for speciality area.

\section{- Principles of care provision}

Knowledge and skills in addition to those acquired for practice as a registered nurse.

\section{- Health promotion}

Knowledge and skills in addition to those acquired for practice as a registered nurse.

- Impact of national and local health and social policies that affect speciality area;

- Agencies, Governmental and NGOs providing services in speciality area;

- National and local health promotion;

- Knowledge of tools of health promotion and prevention strategies; 
- Approaches to provide timely information and education relating to disease to patients and their families on treatment and effects across the continuum;

- Disease-related changes that may lead to limitations with regard to cognitive and sensory skills and memory;

- Skills, identification and interpretation of health and social policy, policy formulation, working with policy-makers and advocacy groups related to speciality;

- Coalition building

- Assessment

Knowledge and skills in addition to those acquired for practice as a registered nurse.

- Epidemiology, physiology and pathology and psycho-social aspects relevant to speciality;

- Techniques, tools and guidelines, laboratory investigations and methods to obtain comprehensive data about patient/client's health status necessary to formulate a diagnosis;

- Skills: advanced clinical assessment skills, interviewing and history taking, physical examination, psycho-social status, use of standardised instruments/tools and diagnostic processes, clinical reasoning and judgment, including interpretation of relevant laboratory tests and diagnostic test results.

\section{- Planning}

Knowledge and skills in addition to those acquired for practice as a registered nurse.

- Major treatment modalities, clinical management and care provision specific to speciality;

- Pharmacological knowledge specific to the speciality area;

- Medication and treatment prescription specific to the speciality area;

- Knowledge of the community and service resources specific to speciality;

- Evidence-based data resources relevant to the speciality;

- Nursing practice models;

- Care planning across the continuum;

- Specialised skills; disease management, symptom control, anticipation, prevention and management of complications, lifestyle management and care co-ordination.

\section{- Implementation}

Knowledge and skills in addition to those acquired for practice as a registered nurse.

- Expanded advanced skills specific to the speciality;

- Management of emergencies specific to the speciality.

\section{- Evaluation}

Knowledge and skills in addition to those acquired for practice as a registered nurse.

- Monitoring and evaluation tools specific to the speciality

- Therapeutic communication and interpersonal relationships

Knowledge and skills in addition to those acquired for practice as a registered nurse.

- Dynamics of conflict and negotiations, solutions and mutual acceptability;

- Information technology;

- Skills: supportive communication, conflict resolutions, information technology skills.

- Leadership and Management

Knowledge and skills in addition to those acquired for practice as a registered nurse.

- Knowledge of leadership, management, change, governance, vision, strategies, goals;

- Positive practice environment, policy development and reviewed processes; 
- Skills: Problem identification, strengths, weaknesses or threats (SWOT) analysis, team building, time management, complaint management, translation of policy to practice, staff development and appraisal, committee work and monitoring and evaluation of services.

- Safe environment

Knowledge and skills in addition to those acquired for practice as a registered nurse.

- Knowledge of safety aspects of speciality area, risks, evidence-based protocols designed to reduce adverse effects, concepts, elements and methodologies of risk assessment;

- Skills: Risk assessment and implementation of risk interventions.

- Inter-professional healthcare

Knowledge and skills in addition to those acquired for practice as a registered nurse.

- Partnership and collaboration (practice models)

- Skills: initiate and respond to referrals.

- Delegation and supervision

Knowledge and skills in addition to those acquired for practice as a registered nurse.

- Role definition;

- Skills: consulting skills.

- Enhancement of the profession

Knowledge and skills in addition to those acquired for practice as a registered nurse.

- Policy-making processes;

- Strategies to influence policy;

- Research: identify questions, use research to change practice;

- Skills: do a literature search, give a critical appraisal related to clinical area, write reports, present and advocate for issues relevant to the speciality, lobbying, press releases.

- Quality improvement

Knowledge and skills in addition to those acquired for practice as a registered nurse.

- Systematic reviews and clinical audits;

- Best practice guidelines;

- Skills: do a clinical audit.

- Continuing education

Knowledge and skills in addition to those acquired for practice as a registered nurse.

- Process of self-assessment and peer-review;

- Professional portfolios;

- Performance appraisals.

- Business skills

Develop and manage a private, independent professional practice; Insurance and indemnity issues. 


\section{Annexure 2:}

\section{Framework competencies for advanced specialist nurse/midwifery practice}

Competencies of the advanced nurse/midwife practitioner are demonstrated in all of the following domains. (Royal College of Nurses 2008: 11). The Synergy model is incorporated (UOFS). The Synergy model attempts to match the skills of caregivers with the needs of patients and with the characteristics of any environment. It is divided into three levels of functioning and the Advanced Practitioner should be at the highest level, Level 3.

\section{Domain 1: Clinical judgment (Health assessment, promotion, and management)}

Clinical reasoning, which includes clinical decision-making, critical thinking, and a global grasp of the situation, coupled with nursing skills acquired through a process of integrating education, experiential knowledge, and evidence-based guidelines (Alspach 2006: 7), including:

\section{Health promotion, protection and prevention}

- Assessment of health and education needs;

- $\quad$ Plan and implement a programme/intervention for health promotion;

- Provides health education to prevent disease and disability;

- Develop and use a follow-up system to ensure well being;

- Promote healthy environments and identify environmental health problems.

\section{Assessment and management of illness}

- Comprehensive assessment, analysis and interpretation of health history, physical examination and applied selected special examination of a health problem to make a differential diagnosis;

- Diagnose and manage acute and long-term conditions and monitor the patient's response to illness and treatment;

- Prioritize healthcare problems and intervene in complex, urgent and emergency situations;

- Initiate and institute appropriate diagnostic and therapeutic interventions, protocols and regimes incorporating principles of safety, cost, invasiveness, simplicity, acceptability, adherence and efficacy;

- Formulate action plans that are founded on evidence-based standards of care, as well as scientific rational and practice guidelines;

- Provide counselling, guidance and support regarding health/illness conditions;

- Initiate timely consultancy/referral when scope of nurse is exceeded by a problem;

- Rapid assessment in complex, urgent emergency care.

- Rapid synthesis and prioritizing of historical and immediate derived data to assess unstable and complex health problems;

- Diagnose unstable and complex problems using collaborative consultation with multiprofessionals as indicated by setting, specialist knowledge and experience;

- Plan and implement specific diagnostic and therapeutic interventions to assist persons with unstable, complex healthcare problems to regain their health, in collaboration with the patient, family and team;

- Rapid and continuous evaluation of the changing condition and response to therapeutic intervention.

- $\quad$ Protect and promote health through

- Comprehensive problem-focused health history-taking skills; 
- Critical, diagnostic reasoning and decision-making;

- Comprehensive age-appropriate physical examination;

- Analysis of data to determine health status;

- Formulate a problem and prioritize an appropriate plan of action;

- Assess, diagnose, monitor and manage acute and ongoing healthcare problems.

- Demonstrate knowledge of the patho-physiology of conditions commonly seen in practice;

- Communicate the patients' healthcare status using appropriate terminology, format and technology;

- Apply principles of epidemiology and recognize the demographic patterns of disease for effective intervention;

- Co-ordinate care and programmes using community-based information and resources;

- Apply evidence-based principles in area of practice;

- Applies information on drug regime, side-effects, interactions and effects in area of practice;

- If permitted, prescribe medication safely, effectively and cost-effectively according to protocols;

- Evaluate complementary medicine used by patient for possible interactions;

- Integrate non-drug treatment into a care plan;

- Order, perform and interpret screening and diagnostic tests;

- Evaluate interventions, adjust, revise, consult and refer.

\section{Domain 2: Caring Practices (Nurse/patient relationship)}

The constellation of nursing activities should create a compassionate, supportive, and therapeutic environment for patients and staff, with the aim of promoting comfort and healing and preventing unnecessary suffering. These caring behaviours include, but are not limited to, vigilance, engagement and responsiveness. Caregivers include family and healthcare personnel (Alspach 2006: 7).

- Develop a climate of mutual trust and partnership with patient, carers and family;

- $\quad$ Validate and check findings with patients;

- Create an empowering relationship that acknowledges the needs, knowledge and strengths of the patient and enables self-care;

- Communicate availability, comfort and support;

- Apply principles of emancipatory decision-making to promote behaviour change;

- $\quad$ Promote and facilitate commitment of patients to take responsibility and control over decisions regarding personal, family and community health;

- Evaluate the impact of the status change on the illness/health continuum on the lives of people, families and communities;

- Monitor and reflect on own responses to interaction with the patient or community and use the knowledge to further the therapeutic relationship;

- Preserve dignity through maintaining confidentiality;

- $\quad$ Provide safe transition to another carer if patient needs this.

\section{Domain 3: Facilitator of learning (Education function)}

The ability to facilitate learning for patients and families, nursing staff, other members of the health care team, and community includes both formal and informal facilitation of learning (Alspach 2006: 7).

- $\quad$ Timing

- Assess on-going education needs of patient, carers, families and communities; 
- Using principles of change and behavioural change and assess motivation for change in healthcare;

- Create an environment for education.

- Eliciting

- Elicit information regarding patient's interpretation, perceived barriers and support, and modifiers to learning, learning styles for an appropriate teaching approach, and cultural influences on the learning experience.

- Enabling

- Enable patients through a sensitivity to efforts to take care of own health;

- Enable the acquisition of new skills in increments allowing for relapse through a supportive programme;

- Enable patients to identify, access and use community resources.

- Providing

- Provide and negotiate a plan of care.

- Negotiating

- Monitor the patient's outcomes and educate.

- Mentoring

- Coaching with empathy to remind, support and encourage.

\section{Domain 4: Advocacy/moral agent (Professional role)}

Working on another's behalf and representing concerns of the patient, family and community; serving as a moral agent in identifying and helping to resolve ethical and clinical concerns within and outside the clinical setting (Alspach 2006: 7).

- Develop and implement advanced role function through

- Initiating and publishing evidence-based research;

- Multi-dimensional role function as advanced clinical consultant, co-ordinator coach, advocate administrator, researcher, role-model and leader;

- Interpret and market the role to profession, public, policy-makers;

- Autonomous accountability.

- $\quad$ Direct care

- Meet diverse needs of a multi-cultural nature;

- Use sound judgment in conflicting priorities and needs;

- Build and maintain a therapeutic team for optimal care;

- Obtain specialist care for patients while remaining the primary provider;

- Act as an advocate for patients in a variety of situations;

- Consult with providers, agencies and the public;

- Incorporate current technology in care;

- Use information systems to enhance decision-making for improved care.

- Provide leadership

- Be actively involved in professional societies;

- Evaluate the implication of current health care policies on care and care provision;

- Participate in policy development and legislation that influence advanced practice;

- Evaluate social problems impacting on healthcare outcomes;

- Be actively involved in continuous professional development and keep a record of this; 
- Solve complex ethical problems.

\section{Domain 5: $\quad$ System thinking (Manage and negotiate healthcare delivery system)}

Body of knowledge and tools that allow the nurse/midwife to manage whatever environmental and system resources exist for the patient/family and staff within or across healthcare and nonhealthcare systems (Alspach 2006: 7).

- Managing

- Knowledgeable re advanced practice role and function;

- Individualized care within an integrated system;

- Consider cost of care;

- Know and use organizational processes, business and management strategies;

- Know and apply relevant regulations of care.

- Negotiating

- Collaborative practice: acknowledge others' expertise;

- Risk assessment and effective management;

- Innovative practice with a multi-disciplinary team;

- Participate in community programmes;

- Advocate for, and participate in, policy and planning re environment, culture sensitivity and improved access.

\section{Domain 6: Clinical enquiry (Monitoring and ensuring quality of advanced practice)}

The ongoing process of questioning and evaluating practice and providing informed practice; creating changes through evidence-based practice, research utilization, and experiential knowledge (Alspach 2006: 7).

- Ensure quality

- Incorporate /professional/legal/standards in advanced practice;

- Act ethically in a complex environment;

- Assume accountability for actions;

- Improve care through clinical supervision and self-assessment;

- Collaborate and consult regarding poor outcomes;

- Promote and use an evidence based approach;

- Evaluate and audit outcomes and effectiveness of care;

- Interpret outcomes so as to improve care;

- Maintain competence.

- Monitor quality

- Monitor quality of own practice;

- Seek and participate in peer review;

- Audit care;

- Keep abreast of evidence-based practice.

\section{Domain 7: Cultural diversity (Respect culture and diversity)}

The sensitivity to recognize, appreciate and incorporate differences into the provision of care. Differences may include, but are not limited to, cultural, spiritual, gender, race, ethnicity, lifestyle, socioeconomic, age and values (Alspach 2006: 7). 
- Cultural

- Demonstrate respect for all along the continuum regardless;

- Respect the rights of individuals to choose providers and refuse care;

- Acknowledge own biases and address these effectively;

- Promote diversity and quality;

- Incorporate beliefs and culture in planning of care;

- Provide patient-appropriate education;

- Assess patient resources for care;

- Support marginalized persons to access care.

- Spiritual

- Respect right to express religious beliefs;

- Refer to pastoral care and assist individual and family to meet spiritual needs

\section{Domain 8: Collaboration}

Working with others (e.g., patients. families, healthcare providers) in a way that promotes and encourages each person's contributions toward achieving optimal and realistic patient/family goals. Collaboration involves intra-disciplinary and interdisciplinary work with colleagues and community (Alspach 2006: 7).

- Work collaboratively with health professionals to promote health and good care 